\title{
Female Doctors: Myths and Facts
}

\author{
Bilal Bin Younis
}

The phenomenon of 'doctor bride' is far too real in Pakistan. A quick Google search of "lady doctors in Pakistan" and the suggested searches that Google come up with, provide a sad glimpse into the status of female doctors in our society. I specifically searched the term "lady doctors" in Pakistan instead of female doctors, the former being the more prevalent term, in order to get an insight into the search behavior and search frequency associated with the term. The suggested searches were as follows:

- Pakistani lady doctors mobile numbers

- Pakistani lady doctors for marriage

- Divorced lady doctor

- Lady doctor proposal in Faisalabad

- MBBS lady doctor for marriage

- Marriage proposal for lady doctor

- Matrimonial adds for lady doctor

- MBBS Matrimonial

The 2016 Asian Development Bank report noted that Pakistani women are increasingly pursuing higher education, however only 25 percent possess a university-level education and work outside of their homes. This situation is reflected in a wide range of professions, including medicine. According to the government body that regulates the medical profession, Pakistan Medical and Dental Council (PMDC), more than 70 percent of medical students are women. However, this does not translate into the working doctors' gender ratio. Moreover, statistical data cites this as one doctor for 1709 people in Punjab (Gallop survey 2018). Bear in mind, this figure accounts for registered doctors, as opposed to practicing doctors, against the population. It is highly plausible hence, that the real figure will paint a far worse picture.

A glance at the listed Google search suggests that marriage is the direct derivative of an MBBS degree. However, while that is devastating and would make for a sensational article, that is not our only problem and the time has come to shift our focus to other relevant issues, those which we can directly address and attempt to fix. The debate regarding the allocation of seats for medical students and whether female students should be allocated fixed seats in medical college was raised by the PMDC in 2014 in an attempt to guarantee more doctors in the work field. The plea was challenged in the court of justice by the female body of students and the human rights activists and thereafter, rejected. However, the solution offered by the PMDC is highly unlikely to work, even if implemented. This is because it discounts merit. Would we rather have less capable doctors working in hospitals than non-working doctors? If phrased as such, the answer is yes. And therein lies the problem, we have been accustomed to think that those are our only two options. We establish that there is a problem but as for the causes of that problem, we blame it on social factors, accept that it cannot be rectified, and call it a day. There are other reasons behind female doctors not being able to work in medical field. These problems we must address. All the same, these are the very real and practical issues women doctors are faced with. These include extensive duty hours, lack of private rooms for breastfeeding, day-care facilities and child-friendly environment for doctors who are mothers. As for women doctors who are married and without children, extensive duty hours as well as rude and sometimes hostile behavior of patients remain very pertinent issues. 
Hence, there is a third option available to fix our problem i.e. collectively reform work policies and environments to suit the schedules and requirement of female doctors. In other words, introduce flexibility and facilitation in our systems. A step further would be to introduce telemedicine to accommodate both male and female doctors into working towards their potential by eliminating the predicament posed by geographical restrictions. By doing so, we may become one step closer towards tackling two problems at once, prevent the merit bar of entrance from being lowered, improve female to male doctor ratio and the patient to doctor ratio. In conclusion, these solutions are rooted in a problem, the degree of which is still unclear. Considering this problem, we are still in the dark as all we have instead of hard facts are a collection of estimates and observations. In order to gain a better insight, we must not ignore the importance of research. While we should begin reforms to accommodate women doctors, we must also conduct extensive first hand research so we may be able to better understand the ground realities at hand and come up with even more productive and effective reforms.

\section{Corresponding Author:}

Prof. Dr. Bilal Bin Younis

Chief Editor, JSHMDC

Professor of Medicine

Sakina Institute of Diabetes \& Endocrinology Research (SiDER),

Shalamar Hospital/ Shalamar Medical

\& Dental College, Lahore.

Email address: bilalbin@gmail.com 